\title{
Recognizing Hand Gestures of a Dancer
}

\author{
Divya Hariharan $^{1}$, Tinku Acharya ${ }^{2}$, and Sushmita Mitra ${ }^{1}$ \\ 1 Machine Intelligence Unit, Indian Statistical Institute, Kolkata 700 108, India \\ sushmita@isical.ac.in \\ 2 Videonetics Technology Private Limited, Kolkata, India \\ tinku. acharya@gmail.com
}

\begin{abstract}
A new and simple two-level decision making system has been designed for performing scale-, translation- and rotation-invariant recognition of various single-hand gestures of a dancer. The orientation filter is used at the first-level to generate a feature vector that is able to distinguish between several gestures. At the second-level the silhouette of the different gestures is extracted, followed by the generation of the corresponding skeleton and the evaluation of the gradients at its end points. These gradients constitute the second feature set, for recognizing those gestures which remain to be identified at the first-level. An application has been provided in the domain of single-hand gestures of Bharatanatyam, an Indian classical dance form.
\end{abstract}

Keywords: Gesture recognition, feature extraction, skeleton matching, orientation histogram.

\section{Introduction}

The term Indian Classical Dance comprises all the art forms of the Natyashastra written by the ancient musicologist Sage Bharata. The Natyashastra confers classical status to eight Indian dances, of which Bharatanatyam is one of the oldest. A popular interpretation of the name is

BHAva (expression) + RAga (musical mode) + TAla (rhythm) + NATYAM (dance) $=$ BHARATANATYAM.

A distinctive feature of Bharatanatyam Dance is the use of expressive hand gestures as a way of communication. Hastas [3] refers to a variety of hand symbols that a dancer can use. Hastas can be broadly classified into two categories, viz., Asamyukta Hastas (Single hand gestures) and Samyukta Hastas (Double hand gestures). There exist 28 Asamyukta Hastas and 24 Samyukta Hastas.

The objective of this research is to develop a suitable prototype for the recognition of the 28 Asamyukta Hastas of Bharatanatyam in a two dimensional space, using image processing techniques [1. We aim to make the computer act as a teacher to correct the dance gestures, for the purpose of promoting e-learning of the nuances of Bharatanatyam across the world.

A major difficulty is associated with the rotation and scaling involved. For example, a gesture image rotated to any degree or scaled to any level should 
represent the same gesture. Orientation filters [2] have been used in various image processing and vision tasks, by applying filters of arbitrary orientation and phase. Typically a few filters, corresponding to a few angles, are employed and the intermediate responses are interpolated. With a correct filter set and interpolation rule, it becomes possible to evaluate a filter of any arbitrary orientation.

In this article we use the orientation filter at the first-level to generate a feature vector for distinguishing between different gestures. At the second-level the silhouette of the different gestures is extracted, followed by the generation of the corresponding skeleton and the evaluation of the gradients at its end points. This constitutes the second feature set, for recognizing those gestures which remain to be identified at the first-level. The rest of the paper is organized as follows. Section 2 describes the generation of the feature vector at the two levels, comprising computation of the orientation histogram and the gradients at the extremities of the hand. The experimental results are provided in detail in Section 3. Finally, Section 4 concludes the article.

\section{Feature Extraction}

The preprocessing of the hand gesture consists of detecting the skin color in the image, and cropping the hand region in order to avoid unnecessary details in the background. The images are then resized to $240 \times 240$ and converted to gray scale. The next step is the feature extraction procedure. A suitable feature vector which is invariant to translation, rotation, scaling and reflection needs to be chosen for the purpose of distinguishing between the different gestures.

\subsection{Edge Orientation Histogram}

Considering the aspects of translation and scaling invariance, the orientation histogram [2] was found to be a useful feature component. Here these constitute histograms of local orientation of the hand gesture. The orientation histograms are robust to illumination changes, and are simple and fast to compute.

Local orientation is obtained by the use of steerable filters, in which a filter of arbitrary orientation is synthesized as a linear combination of a set of "basis filters". A set of 36 one-dimensional Gaussian steerable filters and their first order derivatives have been used for extracting the local edge orientation properties of the hand gesture for every $10^{\circ}$, ranging from $0^{\circ}$ to $350^{\circ}$. The gesture image $I$, filtered at an arbitrary orientation and convolved with the filters, can be synthesized for the oriented filter response

$$
R_{1}^{\theta}=\left(G_{1}^{0^{\circ}} * I\right) \cos \theta+\left(G_{1}^{90^{\circ}} * I\right) \sin \theta,
$$

where $*$ represents the convolution operator, and $G_{1}^{\theta}$ is considered at an arbitrary orientation $\theta$.

In order to enhance the ability of the edge orientation histogram in recognizing the rotated gestures, the direction of maximum local orientation of every gesture is found. Accordingly the original gesture image is rotated in such a manner that 


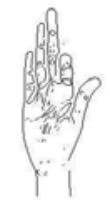

(i)

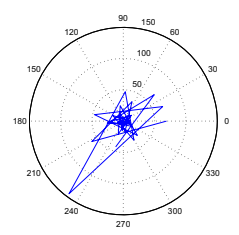

(v)

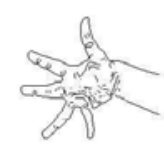

(ii)

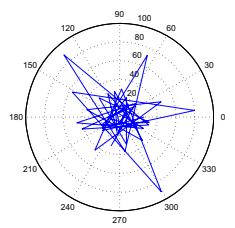

(vi)

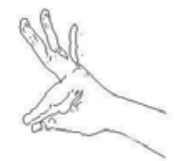

(iii)

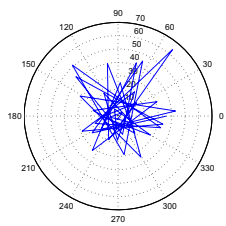

(vii)

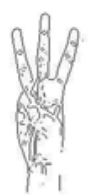

(iv)

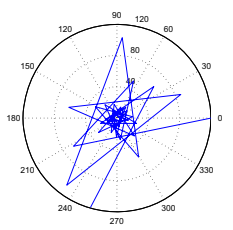

(viii)

Fig. 1. Edges of sample single-hand Bharatanatyam gestures. (i) Aarala, (ii) Alapadma, (iii) Hamsaye, and (iv) Trishula. Polar plots of corresponding edge orientation histograms of gestures (v) Aarala, (vi) Alapadma, (vii) Hamsaye, and (viii) Trishula.

the maximum orientation is obtained at $180^{\circ}$. The edges of the hand gesture are extracted using the Laplacian of Gaussians (LOG) [1], and the image is multiplied with the filter response for every value of $\theta$. Fig. 1 illustrates the polar plots of the edge orientation histogram corresponding to the edges from the gestures "Aarala", "Alapadma", "Hamsaye" and "Trishula".

\subsection{Gradients at Corner Points of Skeleton}

Those gestures that cannot be recognized at the first-level, by the use of edge orientation histograms, are processed further at a second-level. It is known that the end points of a skeleton correspond to a change of curvature. Refined categorization is next made, using the gradients at the extremities of the skeleton

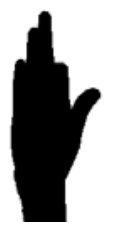

(i)

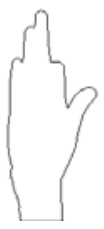

(ii)

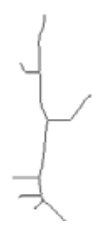

(iii)

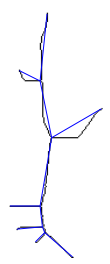

(iv)

Fig. 2. Sample gesture Aarala. (i) Silhouette, (ii) Boundary, (iii) Skeleton, and (iv) Connectivity graph. 
as a new set of features. A given gray scale image is initially binarized in a uniform manner over hand gestures of different people. The boundary is extracted from the binary image, followed by the flood-fill operation to generate a uniform silhouette. This is depicted in Fig. 2(i) for a sample dance gesture "Aarala".

The skeleton [Fig. 2(iii)] is extracted by the morphological operation of thinning. Typically it consists of a number of branch points and end points, connected by curve segments. These points are considered to be the nodes of a graph, such that the skeletal curve segments form the edges. This graph [Fig. 2(iv)] is called the connectivity graph of the skeleton. It provides topological information about the hand gesture.

Matching of the connectivity graphs, based on their topologies and geometric features, provides a distance measure for determining the similarity (or dissimilarity) between the different shapes. The adjacency matrix of the connectivity graph is constructed. The degree of every node in the graph is computed and assigned as its weight. Subsequently, a depth-first traversal sequence is constructed for the connectivity graph starting from one end point (typically, the leftmost point). Preference is provided to a node with a lower weight during the traversal. Thereby, the number of backtracking sequences gets reduced.

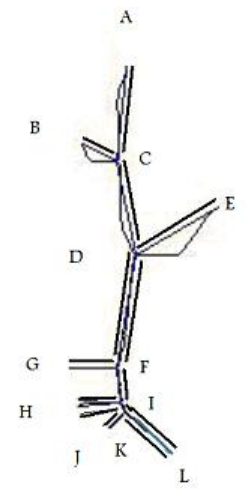

Fig. 3. Depth-first traversal in edge connectivity graph of Aarala

The thick black lines in Fig. 3 indicate the depth-first traversal sequence of the connectivity graph of Fig. 2(iv). The sequence obtained is expressed as

$G \rightarrow F \rightarrow I \rightarrow H \rightarrow I \rightarrow K \rightarrow J \rightarrow K \rightarrow L \rightarrow K \rightarrow I \rightarrow F \rightarrow D \rightarrow E \rightarrow D \rightarrow$ $C \rightarrow B \rightarrow C \rightarrow A$.

Repetitions of nodes correspond to the backtracking of edges during traversal of the graph.

For every end point in the skeleton, the nearest boundary point is obtained. Without loss of generality, it can be inferred that this nearest boundary point will be either a finger tip or a point in the hand gesture where the curvature change is large. Hence the gradient at these points will significantly vary over the different gestures. For the branch points, this value is assigned to be zero in 
order to avoid unnecessary weights due to backtracking sequences. The values of gradients are then substituted for the nodes, in the depth-first traversal sequence. This sequence of gradients and zeros forms the feature vector for the second-level of recognition.

\section{Experimental Results}

The gesture vocabulary was built by capturing ten images of each of the 28 single-hand gestures of Bharatanatyam from the hands of three different people, using a seven megapixel camera. These ten images were translated, rotated, scaled and reflected versions of a single gesture. Hence every image is unique. Out of the ten images, eight were randomly selected for training the system, while the remaining two were kept for testing. This amounted to a total of 224 training images. The test images were identified based on the closest match to the learned examples (prototypes) in terms of Euclidean distance. In the firstlevel, orientation histogram was used for feature extraction. In the second-level a shape-based skeleton matching was used, with the gradients at the corner points being used as a new set of features.

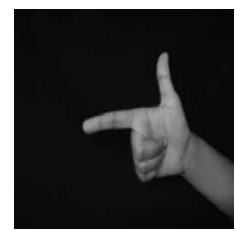

(i)

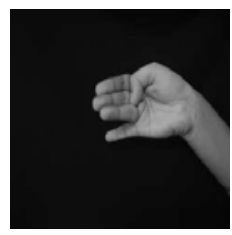

(ii)

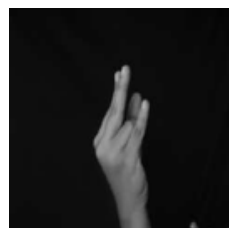

(iii)

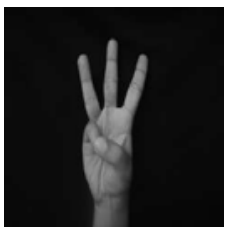

(iv)

Fig. 4. Distinctly identifiable gestures at the first-level. (i) Chandrakala, (ii) Chatura, (iii) Kangula, and (iv) Trishula.

Application of the edge orientation histogram resulted in the correct identification of four gestures, which were found to be distinctly unique from the others. These are Chandrakala, Chatura, Kangula and Trishula, as shown in Fig. 4. The remaining 24 gestures could be grouped into three classes, as indicated by the three rows of Fig. 5. These 24 images were used for subsequent processing at the second-level, as described below.

Shape-based skeleton matching was next used, with the set of gradients at the corner points in the skeleton serving as the new set of features for the remaining 192 (= $224-32)$ training images. The skeleton of the test image of a hand gesture was first obtained, and its connectivity graph generated. Then a depthfirst traversal sequence with the gradient values was computed, for use as the feature vector. The left-most point of a skeleton image was generally considered as the starting point of the depth-first traversal for every gesture. Since all gestures are rotated to give a maximum orientation at $180^{\circ}$, therefore it is safe 


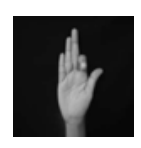

(i)

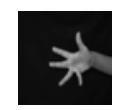

(viii)

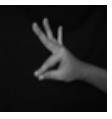

(xvii)

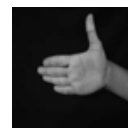

(ii)

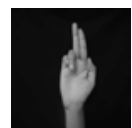

(iii)

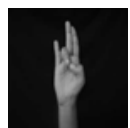

(iv)

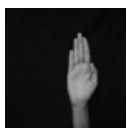

(v)

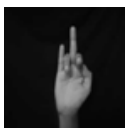

(vi)
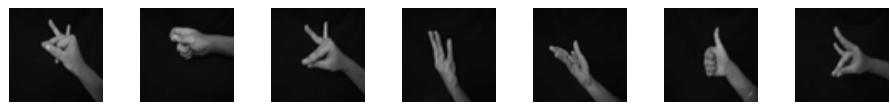

(vii)

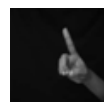

(ix)

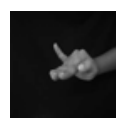

(x)

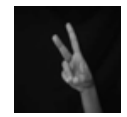

(xi)

(xii)
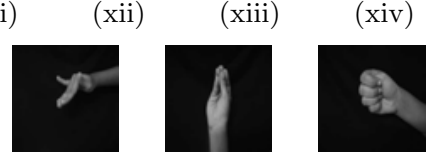

(xv)

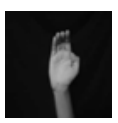

(xvi)

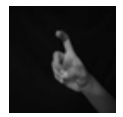

(xx)

(xxi)

(xxii)

(xxiii)

Fig. 5. Three groups of gestures at first-level. Class 1 with (i) Aarala, (ii) Ardhachandra, (iii) Ardhapataka, (iv) Mayura, (v) Pataka, (vi) Shukatunda, (vii) Tripataka. Class 2 with (viii) Alapadma, (ix) Bhramara, (x) Kapitta, (xi) Katakamukha, (xii) Padmakosha, (xiii) Sandamsha, (xiv) Shikara, (xv) Simhamukha, and (xvi) Suchi. Class 3 with (xvii) Hamsaye, (xviii) Hansapakshika, (xix) Kartarimukha, (xx) Mrigashirsa, (xxi) Mukula, (xxii) Mushti, (xxiii) Sarpashirsa, and (xxiv) Tamrachuda.

to assume that for the same gesture the starting point will be the same. The matching of two shapes now reduces to the problem of matching two feature sequences.

Let $V$ and $U$ be two such sequences of lengths $n$ and $m$ respectively. Using concepts from dynamic programming, a cost function $\operatorname{Cost}(i, j)$ is defined as the cost of matching the $i$ th element $V_{i}$ of the first sequence with the $j$ th element $U_{j}$ of the second sequence. It is defined as $\operatorname{Cost}(i, j)=$

$\min \{\operatorname{Cost}(i, j-1), \operatorname{Cost}(i-1, j), \operatorname{Cost}(i-1, j-1)\}+W \operatorname{eight}\left(V_{i}\right) \times V_{i}-W \operatorname{eight}\left(U_{j}\right) \times U_{j}$.

In order to match the sequences $V$ and $U$, one needs to compute the cost over their entire length. It is evident that the value of the cost function determines the similarity (or dissimilarity) between the shapes of different hand gestures. There are, however, possibilities of error due to the irregularities in the skeleton structure from the hands of different people with different textural properties.

After the second-level of processing only four of the gesture types remained incorrectly recognized. These images are presented in Fig. 6. with the upper row indicating the misclassified gesture and the lower depicting the corresponding category with which it has been mistaken. It can be easily observed that each of these image pairs, viz. those in lying in the same column of the figure, are highly similar to the human eye. Hence the performance of the designed system, on the whole, can be claimed to be reasonably good. 


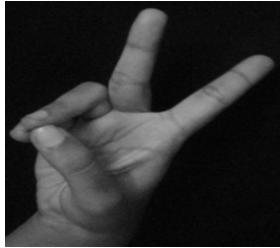

(i)

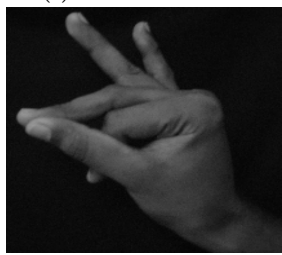

( v)

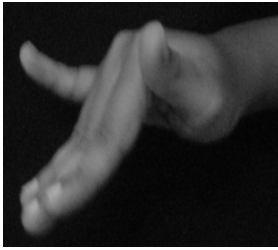

(ii)

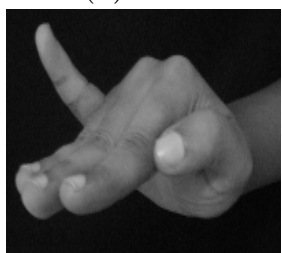

(vi)

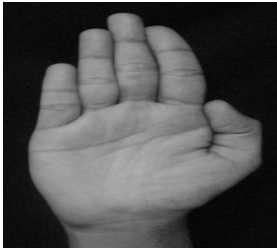

(iii)

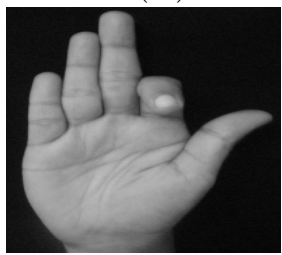

(vii)

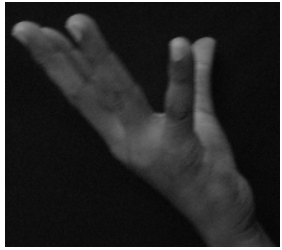

(iv)

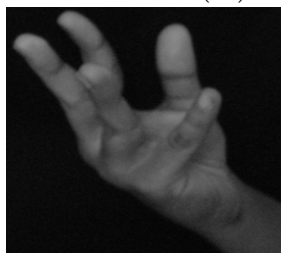

(viii)

Fig. 6. Dance gestures still misclassified at the second-level. (i) Kartarimukha, (ii) Bhramara, (iii) Mrigashirsa, and (iv) Hansapakshika, were mistaken with (v) Pataka, (vi) Aarala, (vii) Sandamsha, and (viii) Padmakosha, respectively.

\section{Conclusion}

E-learning would be a very practical, simple and cost-effective mode of imparting training in the nuances of traditional dance across the globe, thereby assimilating the cultural divide between the Orient and the West. We aim to make the computer act as a teacher to correct the dance gestures for the purpose of promoting classical Indian dance across the world. A simple and new twolevel decision making system has been designed for recognizing the gestures of Bharatanatyam, a well-known Indian classical dance form. It has been shown to be scale-, translation- and rotation-invariant while recognizing various singlehand gestures of a dancer.

Acknowledgement. This research was carried out while Ms. D. Hariharan was in ISI on an IASc-INSA-NASI summer fellowship.

\section{References}

1. Acharya, T., Ray, A.K.: Image Processing Principles and Applications. John Wiley, New Jersey (2005)

2. Freeman, W.T., Roth, M.: Orientation histograms for hand gesture recognition. In: Proceedings of IEEE International Workshop on Automatic Face and Gesture Recognition, pp. 296-301. IEEE, Los Alamitos (1994)

3. Verma, M.: Bharatanatyam: Origin, Styles and Techniques, chap, pp. 295-315. Abhishek Publishers, Hastas (2009) 